\begin{tabular}{|c|l|}
\hline Title & $\begin{array}{l}\text { Spectral and Transient Luminescence Measurements on GaSb/A IGaSb Quantum Wells Grown on GaSb/GaAs } \\
\text { Heterojunctions with and without Interfacial Misfit A rrays }\end{array}$ \\
\hline Author(s) & Jahan, Nahid A .; A hirwar, Pankaj; Rotter, Thomas J.; Bal akrishnan, Ganesh; Kumano, Hidekazu; Suemune, Ikuo \\
\hline Citation & $\begin{array}{l}\text { Japanese Journal of A pplied Physics, 52, 022101 } \\
\text { https://doi.org/10.7567/JAP.52.022101 }\end{array}$ \\
\hline Issue Date & 2013-01 \\
\hline Doc URL & http://hdl.handle.net/2115/52473 \\
\hline Rights & ○ 2013 The Japan Society of A pplied Physics \\
\hline Type & article (author version) \\
\hline File Information & RP120665.pdf \\
\hline
\end{tabular}

Instructions for use 


\title{
Spectral and Transient Luminescence Measurements on GaSb/AlGaSb Quantum Wells Grown on GaSb/GaAs Heterojunctions with and without Interfacial Misfit Arrays
}

\author{
Nahid A. Jahan ${ }^{1,2^{*}}$, Pankaj Ahirwar ${ }^{3}$, Thomas J. Rotter ${ }^{3}$, Ganesh Balakrishnan ${ }^{3}$, Hidekazu \\ Kumano $^{1}$, and Ikuo Suemune ${ }^{1}$ \\ ${ }^{1}$ Research Institute for Electronic Science, Hokkaido University, Sapporo 001-0021, Japan \\ ${ }^{2}$ Graduate School of Information Science and Technology, Hokkaido University, Sapporo 060-0814, \\ Japan \\ ${ }^{3}$ Center for High Technology Materials, University of New Mexico, 1313 Goddard SE Albuquerque, \\ NM 87106, U.S.A. \\ *E-mail: nahid@es.hokudai.ac.jp
}

\begin{abstract}
Growth of $90^{\circ}$ interfacial-misfit-dislocation (IMF) array at heterointerfaces offers low dislocation densities in highly mismatched heterostructures such as GaSb/GaAs. We investigated time-integrated and time-resolved photoluminescence (PL) properties of a $\mathrm{GaSb} / \mathrm{AlGaSb}$ quantum well (QW) structure grown on (001) GaAs substrate with and without IMF array at the GaSb-buffer/GaAs interface. Our observation reveals that the lowtemperature PL from the QW with IMF is twice more intense than that of the QW without IMF, indicating higher quantum efficiency with IMF. The QW with IMF also exhibited the band filling effect at higher excitation power revealed from the spectrally resolved PL decay measurements. These results are the indication of subdued dislocation density with the IMF growth mode. Our PL measurement results along with supportive band-structure calculation of the GaSb/AlGaSb QW show that the luminescence efficiency of the present QW structure is limited by the hole leakage at elevated temperature. Therefore the IMF effect will be more clearly demonstrated by replacing the heterostructure with the one with higher band-offsets.
\end{abstract}




\section{Introduction}

GaSb-based heterostructures have gained a lot of scientific interest owing to narrow band-gap properties $^{1)}$ and high carrier mobility, ${ }^{2-4)}$ which make them suitable candidates for longwavelength light-emitting devices, ${ }^{5-8)}$ high-speed detectors, ${ }^{9)}$ and low-power consumption devices. A wide range of applications such as thermo-photovoltaic (TPV) devices, ${ }^{10,11)}$ medical diagnostics, military scene projection, detection of gas pollution, ${ }^{12,13)}$ as well as the new era of optical-fiber-based communications have been accomplished by the effectual use of antimony-based detectors and emitters.

To comply with the economical demand and also to exploit advanced device functionality with optimum characteristics, it is attractive and challenging to grow $\mathrm{GaSb}$ on a desirable matrix such as on inexpensive and well-established GaAs or Si substrates rather than on native substrates. Despite the considerable interest, the growth of GaSb epilayers on GaAs is fundamentally difficult due to the large (7.8\%) lattice mismatch, resulting in structural defects such as threading dislocations. ${ }^{14,15)}$ Innumerous studies have been carried out to minimize the strain and defects in the growth of highly lattice-mismatched materials by employing lateral overgrowth and thick metamorphic buffer. ${ }^{16,17)}$ Recently, the periodic interfacial misfit dislocation (IMF) growth mode has been demonstrated distinctively ${ }^{18-24)}$ as an alternative approach to accommodate the large mismatch and to offer high-quality bufferless $\mathrm{GaSb}$ on GaAs and related materials. In the IMF growth mode, about $98.5 \%$ of the strain between the epilayer and substrate can be relieved by the formation of a two-dimensional misfit array consisting of $90^{\circ}$ dislocations at the $\mathrm{GaSb} / \mathrm{GaAs}$ interface, where the permissible propagation direction is along the [110] and [-110] directions. ${ }^{18)}$ This IMF is formed by the periodic skipping of atomic bonds which results in a highly periodic array of Ga-dangling bonds localized at the $\mathrm{GaSb} / \mathrm{GaAs}$ interface. ${ }^{21)}$ The misfit periodicity is measured to be 5.56 $\mathrm{nm}$ and corresponds to exactly $13 \mathrm{GaSb}$ lattice sites and $14 \mathrm{GaAs}$ lattice sites. Thus, every $14^{\text {th }} \mathrm{Ga}$ atom has dangling bonds. In this way strain remains localized at the interface rather than propagating in the vertical direction and thus the deleterious vertically propagating threading dislocations are prohibited. ${ }^{18)}$

The IMF growth mode has been applied to GaSb on GaAs, ${ }^{18)} \mathrm{GaSb}$ quantum dots (QDs) on $\mathrm{GaAs},{ }^{19,20)} \mathrm{GaAs}$ on $\mathrm{GaSb},{ }^{22)}$ and $\mathrm{AlSb}$ on $\mathrm{Si}^{24)}$ and mainly the interface structural properties have been discussed in detail based on transmission electron microscopic observations. The effect of the interfacial states on the electronic properties ${ }^{23)}$ and the optical properties $^{19,20)}$ has been also studied previously. This includes the comparison of the 
photoluminescence (PL) properties of GaSb QDs grown on GaAs by the Stranski-Krastanov and IMF growth modes. Although both ensembles showed room temperature PL, ${ }^{19)}$ the effect of the IMF growth mode on the improvement of the optical properties was not clear. Therefore a more comprehensive study is required to understand the effect and influence of the interfacial misfits on the optical and carrier-thermo-dynamical properties of semiconductor heterostructures. In this paper, we prepared two GaSb/AlGaSb quantum well (QW) samples grown on (001) GaAs substrates. The two samples have the same structures except for the GaSb buffer and GaAs substrate heterointerface. One sample was grown with the IMF growth mode (hereafter designated as "QW-IMF") and the other was grown without IMF ("QW-No-IMF"). We show the measured results of the detailed optical properties such as the luminescence spectra, the luminescence quantum efficiencies, and the recombination lifetimes. We discuss the IMF effect by the comparison of the two samples from the viewpoints of the temperature and excitation-power dependences of these optical properties.

\section{Sample Preparation and Measurement Setup}

We studied two GaSb/ $\mathrm{Al}_{0.5} \mathrm{Ga}_{0.5} \mathrm{Sb} \mathrm{QW}$ samples grown on (001) GaAs substrates by molecular-beam epitaxy (MBE). One sample is grown by introducing the IMF arrays at the GaSb-buffer/GaAs heterointerface (QW-IMF), the details of which are presented distinctively elsewhere. ${ }^{18)}$ The other sample is grown directly on GaAs without the formation of IMF at the interface (QW-No-IMF). The key issue for the difference of the two samples is that GaSb buffer is grown on the Ga-stabilized GaAs surface in the former case and it is grown on the As-stabilized surface in the latter case. The schematic illustration of these sample structures of QW-IMF and QW-No-IMF is shown in Figs. 1(a) and 1(b), respectively. For both samples, the GaSb QW of $8 \mathrm{~nm}$ thickness is sandwiched by the $50 \mathrm{~nm}$ thick $\mathrm{Al}_{0.50} \mathrm{Ga}_{0.50} \mathrm{Sb}$ barrier layers and is capped by the $10 \mathrm{~nm}$ thick GaSb layer on top. Concerning the structural difference of the samples of QW-IMF and QW-No-IMF, the cross-sectional transmission electron micrograph images of the interface of $\mathrm{GaSb} / \mathrm{GaAs}$ with IMF array and the interface when grown without IMF growth mode can be found in the related work. ${ }^{25)}$

Time-integrated PL measurements were performed using non-resonant continuous-wave (CW) excitation at the low average power density of $6 \mathrm{~W} / \mathrm{cm}^{2}$ using a frequency doubled $\mathrm{Nd}$ :YAG laser emitting at $2.33 \mathrm{eV}(532 \mathrm{~nm})$. For the temperature dependent PL spectroscopy a $75-\mathrm{cm}$ monochromator with a 300 groves/mm grating and a liquid-Nitrogen-cooled InGaAs photodiode array detector were used. 
Time-resolved PL spectroscopy was performed to investigate the temperature dependent carrier dynamics in the QWs. The $\sim 5$-ps optical pulses generated by a mode-locked Ti:Sapphire laser tuned at $1.59 \mathrm{eV}(780 \mathrm{~nm})$ with a repetition rate of $76 \mathrm{MHz}$ were used for the excitation. A near-infrared streak camera (Hamamatsu C11293-01) combined with a 150groves $/ \mathrm{mm}$ monochromator was used for the detection of the time-resolved PL spectra with 20-ps time resolution.

\section{Results and Discussion}

The two QW samples with and without IMF showed qualitatively similar properties. Therefore the measured results on the QW-IMF sample will be shown first and then the comparison with the QW-No-IMF sample will be discussed quantitatively.

\subsection{PL characterization of QW-IMF sample}

\subsubsection{Time-integrate PL measurements}

The temperature-dependent time-integrated PL measurements were performed on the QWIMF under the low excitation power of $6 \mathrm{~W} / \mathrm{cm}^{2}$ and the measured spectra are shown in Fig. 2(a). The PL emission peak was observed at the photon energy of $878 \mathrm{meV}$ (1412 nm) at 10 $\mathrm{K}$ and the full width at half maximum was $23 \mathrm{meV}$. In the temperature range between 40 and $80 \mathrm{~K}$, the PL emission peak showed an anomalous blue shift as shown by the close circles in Fig. 2(b). The measured temperature dependence was fitted using the well-known Varshni empirical expression ${ }^{26)}$

$$
E(T)=E_{0}-\frac{\alpha T^{2}}{\beta+T}
$$

where $E_{0}$ is the energy gap at $0 \mathrm{~K}, T$ is the temperature in $\mathrm{K}, \alpha$ and $\beta$ are the fitting parameters. The dashed lines are the fittings using the Varshni parameters of $\alpha=0.42 \mathrm{meV} / \mathrm{K}$ and $\beta=140 \mathrm{~K}$ reported on $\mathrm{GaSb}^{27)}$ and the two $E_{0}$ values of 878 and $893 \mathrm{meV}$. The temperature dependence below $40 \mathrm{~K}$ and above $80 \mathrm{~K}$ is well fitted with Eq. (1) and follows the typical temperature dependence of the semiconductor band gap. Therefore the observed transient blue-shift is attributed to the localization of excitons in the states formed by the QW interface fluctuation at the lower temperature and their thermal delocalization at the higher temperature. $^{28)}$

The spectrally integrated PL intensity is plotted in Fig. 2(c) with the closed circles as a function of inverse temperature. The solid line is the fitting to the experimental data using the Arrhenius equation, 


$$
I(T)=\frac{I_{0}}{1+B \exp \left(-\frac{E_{\mathrm{a}}}{k T}\right)},
$$

where $I_{0}$ is the integrated PL intensity at $0 \mathrm{~K}, E_{\mathrm{a}}$ is the thermal activation energy, $k$ is the Boltzmann constant, and $T$ is the sample temperature in $K . B$ is the dimensionless coefficient which is defined simply as the ratio of carrier capture time and carrier escape time related to non-radiative recombination processes. ${ }^{29)}$ For the QW-IMF, the best fit of Eq. (2) yields the activation energy of $E_{\mathrm{a}}=17 \pm 3 \mathrm{meV}$ and the corresponding coefficient of $B=303 \pm 43$.

\subsubsection{Time-resolved PL measurements}

To investigate carrier dynamics and thermally activated escape processes in the QW structures, time-resolved spectroscopy generally gives us deeper insight. Transient PL measurements were performed as a function of excitation laser power and environmental temperature. The measured results at $4 \mathrm{~K}$ are exemplarily shown for the average excitation powers of 30, 1550, and $3000 \mathrm{~W} / \mathrm{cm}^{2}$ in Fig. 3(a). The transient PL intensity is given with the spectral integration and the solid line is the fitting with the double exponential function of $I(\mathrm{t})=I_{\text {dark }}+A_{1} \exp \left(-\mathrm{t} / \tau_{1}\right)+A_{2} \exp \left(-\mathrm{t} / \tau_{2}\right)$, where $I_{\text {dark }}$ is the dark counts without the pulsed laser excitation. From the PL transient obtained under the low excitation power of $30 \mathrm{~W} / \mathrm{cm}^{2}$, the two time decay constants were determined as $\tau_{1}=220 \pm 6$ ps and $\tau_{2}=1006 \pm 21$ ps with the amplitude ratio of $A_{1} / A_{2}=4.47$. To identify the origin of the two decay constants, the PL transient decay was reproduced with different spectral integrations. The spectral integration in the energy range of 882 to $907 \mathrm{meV}(1367-1405 \mathrm{~nm})$ resulted in the fast decay shown in Fig. 3(b) and was well fitted with the single exponential function with the decay time constant of 217 ps. The spectral integration in the energy range of 859 to $882 \mathrm{meV}$ (1406-1444 nm) resulted in the longer decay shown in Fig. 3(c) and was also well fitted with the single exponential function with the decay time constant of $990 \mathrm{ps}$. These time constants show very good agreement with those estimated with the total spectral integration. This indicates that the fast decay constant originates from the higher-energy excited states of the QW which experience fast energy relaxation and the longer one from the lower-energy ground states.

Similar analyses were performed for all the excitation power dependent PL transients, and the measured decay time constants are summarized in Table I. It is clearly shown that the longer decay time of the ground state remains almost unchanged against the increase of the excitation power. On the other hand the excited-state decay time constant showed the clear 
increase from 220 to 350 ps with the increase of the average excitation power from 30 to $3000 \mathrm{~W} / \mathrm{cm}^{2}$. This is the clear indication of the band filling effect, that is, the increase of the carrier population slows down the energy relaxation from the excited states and therefore the effective lifetime of the excited states is extended.

The temperature-dependent time-resolved PL measurements were also performed under the constant excitation power of $30 \mathrm{~W} / \mathrm{cm}^{2}$, and the measured results are shown in Fig. 4(a). The temperature dependence of the ground state PL decay time constants derived with the method described above is plotted in Fig. 4(b). The decay time constant of 1006 ps the low temperature of $4 \mathrm{~K}$ is drastically reduced for the higher temperature. The observed temperature dependence was fitted with the following equation ${ }^{30)}$

$$
\tau(T)=\frac{\tau_{d}}{1+\left(\tau_{d} / \tau_{e}\right) \exp ^{-E_{a} / k T}}
$$

where $T$ is the temperature, $k$ is the Boltzmann constant, $\tau_{d}(1006 \mathrm{ps})$ is the recombination lifetime at the low temperature limit, $E_{\mathrm{a}}$ is the activation energy, and $\tau_{\mathrm{e}}$ is the escape time of charge carriers from the QW to the barriers. The fitting of the experimental data results in the escape time of $53 \pm 8 \mathrm{ps}$ and the activation energy of $16 \pm 3 \mathrm{meV}$. This activation energy of $16 \mathrm{meV}$ derived from the time-resolved measurements is very close to the activation energy of $17 \mathrm{meV}$ deduced from the temperature dependence of the time-integrated PL measurements shown in Fig. 3(c).

\subsection{PL measurements of QW-No-IMF and comparison to QW-IMF}

In order to study the effect of IMF on the optical quality of QWs grown above the heterointerface, we measured the sample with the same QW structure but without IMF, i.e., the sample QW-No-IMF. The time-integrated and time-resolved PL measurements were performed in the similar manner and we observed qualitatively similar results. Therefore we will focus on the quantitative differences between QW-IMF and QW-No-IMF.

The PL peak of QW-No-IMF at $10 \mathrm{~K}$ under the low excitation power of $6 \mathrm{~W} / \mathrm{cm}^{2}$ was observed at $879 \mathrm{meV}(1410 \mathrm{~nm})$ and is almost the same as that of QW-IMF. The measured temperature dependence showed the blue shift from 40 to $76 \mathrm{~K}$ in the same way as the one shown in Fig. 2(b). The temperature dependence of the time-integrated PL intensity was measured and was fitted in the same way by using Eq. (2), which resulted in the activation energy of $14 \mathrm{meV}$. This is slightly smaller than that of $17 \mathrm{meV}$ for QW-IMF (not displayed here). 
The time-resolved PL measurements performed on the QW-No-IMF also revealed two decay time constants. The PL traces at the power of 30,1550 , and $3000 \mathrm{~W} / \mathrm{cm}^{2}$ are exemplarily displayed in Fig. 5(a) and the extracted decay time constants are summarized in Table I together with those of QW-IMF. The lower-energy ground state showed the slightly longer decay time of about $1.5 \mathrm{~ns}$ and the higher-energy excited states showed the one of around 250 ps. The most important difference is that the excited-state decay time remained constant for the increase of the excitation power unlike QW-IMF. This means that the band filling effect is quenched in the case of QW-No-IMF. This suggests the presence of additional nonradiative recombination process ${ }^{31)}$ or carrier leakage passage in this sample and therefore inferior optical quality.

The temperature-dependent PL transient measurements showed the drastic reduction of the PL decay time with increasing temperature in the same way as shown in Fig. 4. The derived temperature dependent decay time constant is displayed in Fig. 5(b). The activation energy obtained from the fitting of the temperature dependence is found to be $10 \pm 2 \mathrm{meV}$ in this case which is smaller than the value of $16 \mathrm{meV}$ shown in Fig. 4(b) for QW-IMF. The escape time is estimated to be $\tau_{\mathrm{e}}=10 \pm 3.7 \mathrm{ps}$ and the recombination lifetime to be $1590 \pm 20 \mathrm{ps}$.

The comparison of the time-integrated PL spectra of the QW-IMF and QW-No-IMF measured under the same excitation power of $40 \mathrm{~W} / \mathrm{cm}^{2}$ is shown in Fig. 5(c). It is observed that the PL intensity of QW-IMF is twice more intense than that of the QW-No-IMF and the PL peak of QW-IMF is blue-shifted in comparison to that of QW-No-IMF. These differences imply that the quantum efficiency in the QW-IMF sample is increased by the insertion of the strain relief IMF layer at the GaSb-buffer and GaAs heterointerface.

\subsection{Discussion}

From the comparison of the excitation power dependence of the excited-state recombination lifetime and the spectra under the same excitation condition, it is now clear that the QW structure prepared with the IMF growth mode exhibits more clear band filling effect by the reduction of defects that quench photo-generated carriers and therefore the IMF at the $\mathrm{GaSb} / \mathrm{GaAs}$ interface improve the optical quality of the upper-grown semiconductor layers. On the other hand the luminescence from the GaSb/AlGaSb QW is rapidly quenched and the ground-state recombination lifetimes are drastically shortened for the elevated temperature. The mechanism is discussed below based on the above-measured activation energies. 


\subsubsection{Calculation of unstrained QW-IMF band structure based on PL measurements.}

To study the relation of the measured activation energies and the QW structure, the QW subband energies are examined employing the known parameters such as semiconductor energy gaps and effective masses as well as the QW width and the measured optical transition energies. In the case of the QW-IMF, 98.5\% of the strain is reported to be relieved with the insertion of the IMF arrays at the GaSb/GaAs interface. ${ }^{18)}$ Therefore no strain contribution was considered to calculate the GaSb/AlGaSb QW band structure grown on the strainrelieved GaSb buffer layer. The unstrained energy gaps of the GaSb QW, the exciton binding energy of $\mathrm{GaSb}$ and the $\mathrm{Al}_{0.50} \mathrm{Ga}_{0.50} \mathrm{Sb}$ barriers are known or interpolated as $813,1.5$, and $1203 \mathrm{meV}$, respectively, from the reported materials data. ${ }^{32-34)}$

The QW confinement energies were calculated by solving the Schrödinger equation for the $\mathrm{GaSb} / \mathrm{AlGaSb}$ finite potential well. The calculation was repeated by varying the band offset to reproduce the experimentally observed QW emission energy of $878 \mathrm{meV}$ at the low temperature with the calculated ground-state transition energy. In the present $\mathrm{GaSb} / \mathrm{AlGaSb}$ common-anion heterostructures, it is known that the band offsets are localized to the conduction band. Employing the iteration method it is found that the small valance-band offset of $13 \%$ of the energy-gap difference gives the most precise agreement. Our calculated band parameters are summarized in Fig. 6 and the calculated transition energy is given by $E_{T}=E_{g}-E_{\text {exc-binding }}+E_{e}^{1}+E_{H H}^{1}=(813-1.5+57+9) \mathrm{meV}=877.5 \mathrm{meV}$, where, $E_{e}{ }^{1}=57 \mathrm{meV}$ is the ground-state electron confinement energy, $E_{H H}{ }^{1}=9 \mathrm{meV}$ is the ground-state heavy-hole $(\mathrm{HH})$ confinement energy, $E_{\text {exc-binding }}=1.5 \mathrm{meV}$ is the exciton binding energy, and $E_{g}=813$ $\mathrm{meV}$ is the GaSb energy gap. For this calculation the QW width was assumed to be a slightly narrower value of $7.5 \mathrm{~nm}$ to give the best fit to the measured transition energy of $878 \mathrm{meV}$. However, the variation of the valence-band confinement energies for the experimentally determined well thickness of $8 \mathrm{~nm}$ from that of the assumed well thickness of $7.5 \mathrm{~nm}$ is minor with the values of 0.71 and $0.3 \mathrm{meV}$ for the $E_{H H}{ }^{1}$ and $E_{H H}{ }^{2}$, respectively.

Based on the band structures given in Fig. 6, holes experience lower barriers for escaping from the QW. Therefore it will be natural to consider the hole thermal escape to the barriers. Holes will be populated dominantly in the ground state $E_{H H}{ }^{1}$ but will be partially populated in the excited state $E_{H H}{ }^{2}$. The thermal barrier for the holes populated in the excited states is 17 $\mathrm{meV}$ and this is almost equal to the measured thermal activation energy of 17 and $16 \mathrm{meV}$ as shown in Figs. 2(c) and 4(b), respectively. This concludes that the PL quenching at the elevated temperature is due to the hole escape from the GaSb QW sub-bands to the AlGaSb 
barriers. The light-hole sub-bands will also take part in this process. The similar calculation resulted in the ground state light-hole confinement energy of $E_{L H}{ }^{1}=25.1 \mathrm{meV}$ and absence of excited states. This energy is in-between the $E_{H H}{ }^{1}$ and $E_{H H}{ }^{2}$ and does not influence the above discussion.

\subsubsection{Carrier-escape mechanism and comparison between $Q W$-IMF and $Q W-N o-I M F$}

The above discussion indicated that the dominant PL quenching mechanism in the present QW structure is the hole leakage from the valence-band QW energy state to the barriers. Generally speaking, carrier escape mechanisms are not limited to this case. The principal parameter is the ratio of $v=E_{a} / \Delta E,{ }^{35-38)}$ where $E_{\mathrm{a}}$ is the activation energy of the escape process and $\Delta E$ is the energy difference between the transition energy of an emitting area and the neighboring higher energy states. When this ratio, $v$ is equal to 1 , exciton escape to the barrier is dominant. When $v=1 / 2$, the carrier escape is in the form of correlated electron-hole pairs and this mechanism was observed with GaInP/AlGaInP QW, ${ }^{35)}$ InAs/GaAs QD, ${ }^{36)}$ InAs/InP QD, ${ }^{37)}$ and InAs/InGaAlAs QD. ${ }^{38)}$ This mechanism is probable when the band discontinuities both in the conduction and valence bands have relatively large values for carrier confinements. When $v<1 / 2$, single carrier escape mechanism dominates ${ }^{36)}$ and this is applied in the present $\mathrm{GaSb} / \mathrm{AlGaSb}$ case. This is because of the band offset which is localized to the conduction band as is shown in Fig. 6.

From the comparison of the time-resolved PL results between the QW-IMF and QW-NoIMF, the activation energy for PL quenching is $10 \mathrm{meV}$ for QW-No-IMF and is smaller than that of $16 \mathrm{meV}$ for the QW-IMF. The carrier-escape time is $10 \mathrm{ps}$ for QW-No-IMF and is also shorter than that of $53 \mathrm{ps}$ for QW-IMF. These differences suggest that QW-No-IMF includes additional carrier escape path related to residual defects. This implies that the defect densities in the QW-No-IMF are larger. These results conclude that the QW structure grown with the IMF growth mode includes the lower density of optically active defects and therefore demonstrate the effectiveness of the IMF growth mode.

\section{Conclusions}

We investigated GaSb/AlGaSb QW structure grown with and without IMF array at the GaSbbuffer/GaAs interface with time-integrated and time-resolved PL measurements. Our observation revealed that the QW grown with IMF showed brighter luminescence and band filling effect from the spectral measurements. The time-resolved PL measurements also showed the band filling effect for the QW prepared with the IMF growth mode. These results 
clarified that the optically active defect densities are substantially reduced with the IMF growth mode. However the luminescence was swiftly quenched for the elevated temperature. This was attributed to the hole leakage from the GaSb well to the AlGaSb barriers. Therefore the IMF effect will be more clearly demonstrated when a heterostructrure with the higher band offsets is grown with the IMF growth mode.

\section{Acknowledgment}

NAJ acknowledges the financial support via a MEXT scholarship and GCOE-RA-ship.

\section{References}

[1] I. Vurgaftman, J. R. Meyer, and L. R. Ram-Mohan: J. Appl. Phys. 89 (2001) 5815.

[2] S. M. Sze: Semiconductor devices (Wiley, New York, 2002).

[3] B. R. Bennett, M. G. Ancona, J. B. Boos, and B. V. Shanabrook: Appl. Phys. Lett. 91 (2007) 042104.

[4] F. Hatami, N. N. Ledentsov, M. Grundmann, J. Böhrer, F. Heinrichsdorff, M. Beer, D. Bimberg, S. S. Ruvimov, P. Werner, U. Gösele, J. Heydenreich, U. Richter, S. V. Ivanov, B. Ya. Meltser, P. S. Kop'ev, and Zh. I. Alferov: Appl. Phys. Lett. 67 (1995) 656.

[5] H. K. Choi and S. J. Eglash: Appl. Phys. Lett. 59 (1991) 1165.

[6] J. Tatebayashi, A. Jallipalli, M. N. Kutty, S. H. Huang, G. Balakrishnan, L. R. Dawson, and D. L. Huffaker: Appl. Phys. Lett. 91 (2007) 141102.

[7] N. Bertru, A. Baranove, Y. Cuminal, G. Almuneau, F. Genty, A. Joullié, O. Brand, A. Mazuelas, and K. H. Ploog: Semicond. Sci. Technol. 13 (1998) 936.

[8] A. D. Andreev, E. P. O'Reilly, A. R. Adams, and T. Ashley: Appl. Phys. Lett. Appl. Phys. Lett. 78 (2001) 2640.

[9] G. Bougnot and F. J. De Lannoy: Electrochem. Soc. 135 (1988) 1783.

[10] G. W. Charache, P. F. Baldasaro, L. R. Danielson, D. M. DePoy, M. J. Freeman, C. A. Wang, H. K. Choi, D. Z. Garbuzov, R. U. Martinelli, V. Khalfin, S. Saroop, J. M. Borrego, and R. J. Gutmann: J. Appl. Phys. 85 (1999) 2247.

[11] H. K. Choi, C. A. Wang, G. W. Turner, M. J. Manfra, D. L. Spears, G. W. Charache, L. R. Danielson, and D. M. Depoy: Appl. Phys. Lett. 71 (1997) 3758.

[12] J. A. Gupta, P. J. Barrios, J. Lapointe, G. C. Aers, and C. Storey: Appl. Phys. Lett. 95 (2009) 041104. 
[13] J. -C. Nicolas, A. N. Baranov, Y. Cuminal, Y. Rouillard, and C. Alibert: Appl. Opt. 37 (1998) 7906.

[14] Y. K. Noh, Y. J. Hwang, and M. D. Kim: J. Korean Phys. Soc. 50 (2007) 1929.

[15] Y. H. Kim, J. Y. Lee, Y. G. Noh, M. D. Kim, and J. E. Oh: Appl. Phys. Lett. 90 (2007) 241915.

[16] J. B. Rodriguez, L. Cerutti, P. Grech, and E. Tournie: Appl. Phys. Lett. 94 (2009) 061124.

[17] R. J. Malik, J. P. van der Ziel, B. F. Levine, C. G. Bethea, and J. Walker: J. Appl. Phys. 59 (1986) 3909.

[18] S. H. Huang, G. Balakrishnan, A. Khoshakhlagh, A. Jallipalli, L. R. Dawson, and D. L. Huffaker: Appl. Phys. Lett. 88 (2006) 131911.

[19] G. Balakrishnan, J. Tatebayashi, A. Khoshakhlagh, S. H. Huang, A. Jallipalli, L. R. Dawson, and D. L. Huffaker: Appl. Phys. Lett. 89 (2006) 161104.

[20] J. Tatebayashi, A. Khoshakhlagh, S. H. Huang, L. R. Dawson, G. Balakrishnan, and D. L. Huffaker: Appl. Phys. Lett. 89 (2006) 203116.

[21] A. Jallipalli, G. Balakrishnan, S. H. Huang, A. Khoshakhlagh, L. R. Dawson, and D. L. Huffaker: J. Cryst. Growth 303 (2007) 449.

[22] A. Jallipalli, K. Nunna, M. N. Kutty, G. Balakrishnan, L. R. Dawson, and D. L. Huffaker: Appl. Phys. Lett. 95 (2009) 202107.

[23] S. H. Huang, G. Balakrishnan, M. Mehta, A. Khoshakhlagh, L. R. Dawson, and D. L. Huffaker: Appl. Phys. Lett. 90 (2007) 161902.

[24] S. H. Huang, G. Balakrishnan, A. Khoshakhlagh, L. R. Dawson, and D. L. Huffaker: Appl. Phys. Lett. 93 (2008) 071102.

[25] A. Jallipalli, G. Balakrishnan, S. H. Huang, T. J. Rotter, K. Nunna, B. L. Liang, L. R. Dawson, and D. L. Huffaker: Nanoscale Res Lett 4 (2009) 1458.

[26] Y. P. Varshni: Physica (Amsterdam) 34 (1967) 149.

[27] M. Motyka, R. Kudrawiec, J. Misiewicz, M. Hümmer, K. Rößner, T. Lehnhardt, M. Müller, and A. Forche: J. Appl. Phys. 103 (2008) 113514.

[28] W. Z. Shen, S. C. Shen, W. G. Tang, Y. Zhao, and A. Z. Li: J. Appl. Phys. 78 (1995) 5696.

[29] E. C. Le Ru, J. Fack, and R. Murray: Phys. Rev. B 67 (2003) 245318.

[30] G. Rainò, A. Salhi, V. Tasco, R. Intartaglia, R. Cingolani, Y. Rouillard, E. Tournié, and M. De Giorgi: Appl. Phys. Lett. 92 (2008) 101931. 
[31] T. S. Shamirzaev, A. M. Gilinsky, A. K. Kalagin, A. I. Toropov, A. K. Gutakovskii, and K. S. Zhuravlev: Semicond. Sci. Technol. 21 (2006) 527.

[32] M.-C. Wu and C.-C. Chen: J. Appl. Phys. 72 (1992) 4275.

[33] M. Geddo, R. Ferrini, M. Patrini, S. Franchi, A. Baraldi, and R. Magnanini: Appl. Phys. Lett. 73 (1998) 1254.

[34] I. Vurgaftman, J. R. Meyer, and L. R. Ram-Mohan: J. Appl. Phys. 89 (2001) 5815.

[35] P. Michler, A. Hangleiter, M. Moser, M. Geiger, and F. Scholz: Phys. Rev. B 46 (1992) 7280.

[36] W. D. Yang, R. R. Lowe-Webb, H. Lee, and P. C. Sercel: Phys. Rev. B 56 (1997) 13314.

[37] G. Gelinas, A. Lanacer, R. Leonnelli, R. A. Masut, and P. J. Poole: Phys. Rev. B 81 (2010) 235426.

[38] C. Hermannstädter, N. A. Jahan, J.-H. Huh, H. Sasakura, K. Akahane, M. Sasaki, and I. Suemune: New J. Phys. 14 (2012) 023037. 


\section{Figure Captions:}

Fig. 1: (a) Schematic illustration of the $\mathrm{GaSb} / \mathrm{Al}_{0.50} \mathrm{Ga}_{0.50} \mathrm{Sb} \mathrm{QW}$ heterostructure grown on GaAs substrate including the IMF arrays at the interface of $\mathrm{GaSb} / \mathrm{GaAs}$ heterojunction. (b) Schematic illustration of the $\mathrm{GaSb} / \mathrm{Al}_{0.50} \mathrm{Ga}_{0.50} \mathrm{Sb} \mathrm{QW}$ heterostructure directly grown on GaAs substrate without IMF arrays in between the GaSb/GaAs heterojunction.

Fig. 2: (a) The temperature dependent PL spectra of QW-IMF for temperatures between 10 and $200 \mathrm{~K}$. A dotted line is drawn to emphasize the anomalous blue shift between 40 to $80 \mathrm{~K}$ and typical red shift for the temperature above $80 \mathrm{~K}$. (b) Temperature dependent energy peak shifts of QW-IMF. The dashed lines are fitting with the Varshni relation. (c) Temperature dependent integrated PL intensities of QW-IMF. The solid line is fitted by the Arrhenius law and results in the activation energy of $17 \mathrm{meV}$.

Fig. 3: (a) The time-resolved PL transients of QW-IMF measured at $4 \mathrm{~K}$ under the average excitation power of 30,1550 , and $3000 \mathrm{~W} / \mathrm{cm}^{2}$. The transient decay is shown with spectral integration of the PL counts between 1363-1445 nm. The solid lines are the fitting with the double-exponential function. (b) The transient PL trace under the average excitation power of $30 \mathrm{~W} / \mathrm{cm}^{2}$ of QW-IMF obtained by spectrally binning the data between 1367-1405 nm (882-907 meV). The solid line is the fitting with the single-exponential function (c) The transient PL trace under the average excitation power of $30 \mathrm{~W} / \mathrm{cm}^{2}$ of QW-IMF by spectrally binning the data between 1406-1444 $\mathrm{nm}(859-882 \mathrm{meV})$. The solid line is obtained by fitting with the single-exponential function.

Fig. 4: (a) The transient PL traces of QW-IMF measured at the temperature of 4, 25, 45, and 120 K. (b) Temperature dependence of the derived decay time constant and the solid line is the fit using Eq. (3). This results in the activation energy of $16 \mathrm{meV}$.

Fig. 5: (a) The time-resolved PL transients of QW-No-IMF measured at $4 \mathrm{~K}$ using the average excitation power of 30,1550 , and $3000 \mathrm{~W} / \mathrm{cm}^{2}$. The solid lines are the resultant fits using double exponential-function. (b) An activation energy of $10 \mathrm{meV}$ is obtained by fitting the temperature dependent decay time constant of QW-No-IMF using Eq. (3). (c) Timeintegrated PL spectra of QW-IMF and QW-No-IMF under the same excitation power of 40 
W/cm ${ }^{2}$. This shows that the QW-IMF PL is twice more intense and blue-shifted in comparison to that of QW-No-IMF.

Fig. 6: Illustration of calculated band diagram of unstrained $\mathrm{GaSb} / \mathrm{Al}_{0.5} \mathrm{Ga}_{0.5} \mathrm{Sb} \mathrm{QW}$ structure. The physical values given in bold (red) are the input parameters from known data and the values given in italic (blue) were calculated. The measured activation energy of $17 \mathrm{meV}$ corresponds to the energy difference of the $\mathrm{HH}$ excited-state sub-band and the barrier valence-band edge. 
Table I. The list of decay times of QW-IMF and QW-NO-IMF.

\begin{tabular}{|c|c|c|c|c|}
\hline \multirow{4}{*}{$\begin{array}{l}\text { Excitation } \\
\text { power } \\
\left(\mathrm{W} / \mathrm{cm}^{2}\right)\end{array}$} & \multicolumn{4}{|c|}{ Decay time (ps) } \\
\hline & \multicolumn{2}{|c|}{ QW-IMF } & \multicolumn{2}{|c|}{ QW-No-IMF } \\
\hline & $\begin{array}{l}\text { Ground-state } \\
\text { decay time }\end{array}$ & $\begin{array}{l}\text { Excited-state } \\
\text { decay time }\end{array}$ & $\begin{array}{c}\text { Ground-state } \\
\text { decay time }\end{array}$ & $\begin{array}{c}\text { Excited-state } \\
\text { decay time }\end{array}$ \\
\hline & $\begin{array}{l}\text { Spectrally- } \\
\text { integrated } \\
\text { [Spectrally- } \\
\text { resolved] }\end{array}$ & $\begin{array}{l}\text { Spectrally- } \\
\text { integrated } \\
\text { [Spectrally- } \\
\text { resolved] }\end{array}$ & $\begin{array}{l}\text { Spectrally- } \\
\text { integrated }\end{array}$ & $\begin{array}{l}\text { Spectrally- } \\
\text { integrated }\end{array}$ \\
\hline 30 & $\begin{array}{c}1006 \pm 21 \\
{[986 \pm 17]}\end{array}$ & $\begin{array}{c}222 \pm 6 \\
{[217 \pm 8]}\end{array}$ & $1590 \pm 20$ & $250 \pm 8$ \\
\hline 150 & $\begin{array}{c}1000 \pm 14 \\
{[977 \pm 16]}\end{array}$ & $\begin{array}{c}260 \pm 7 \\
{[255 \pm 10]}\end{array}$ & $1530 \pm 27$ & $243 \pm 7$ \\
\hline 750 & $\begin{array}{c}1050 \pm 17 \\
{[993 \pm 10]}\end{array}$ & $\begin{array}{c}310 \pm 7 \\
{[303 \pm 6]}\end{array}$ & $1489 \pm 30$ & $249 \pm 5$ \\
\hline 1550 & $\begin{array}{c}1004 \pm 20 \\
{[980 \pm 17]}\end{array}$ & $\begin{array}{c}330 \pm 10 \\
{[326 \pm 8]}\end{array}$ & $1550 \pm 26$ & $258 \pm 7$ \\
\hline 3000 & $\begin{array}{c}1019 \pm 28 \\
{[988 \pm 18]}\end{array}$ & $\begin{array}{c}351 \pm 8 \\
{[345 \pm 6]}\end{array}$ & $1560 \pm 19$ & $257 \pm 9$ \\
\hline
\end{tabular}


Figure 1 (1 col.)



(a)

\begin{tabular}{|c|}
\hline GaSb cap $5 \mathrm{~nm}$ \\
\hline $\mathrm{Al}_{0.5} \mathbf{G a}_{0.5} \mathrm{Sb} 50 \mathrm{~nm}$ \\
\hline $\mathbf{G a S b ~} 8 \mathrm{~nm}$ \\
\hline $\mathrm{Al}_{0.5} \mathbf{G a}_{0.5} \mathrm{Sb} 50 \mathrm{~nm}$ \\
\hline $\mathbf{G a S b} 100 \mathrm{~nm}$ \\
\hline GaAs SI-Substrate \\
\hline
\end{tabular}

(b) 
Figure 2 (1 col.)
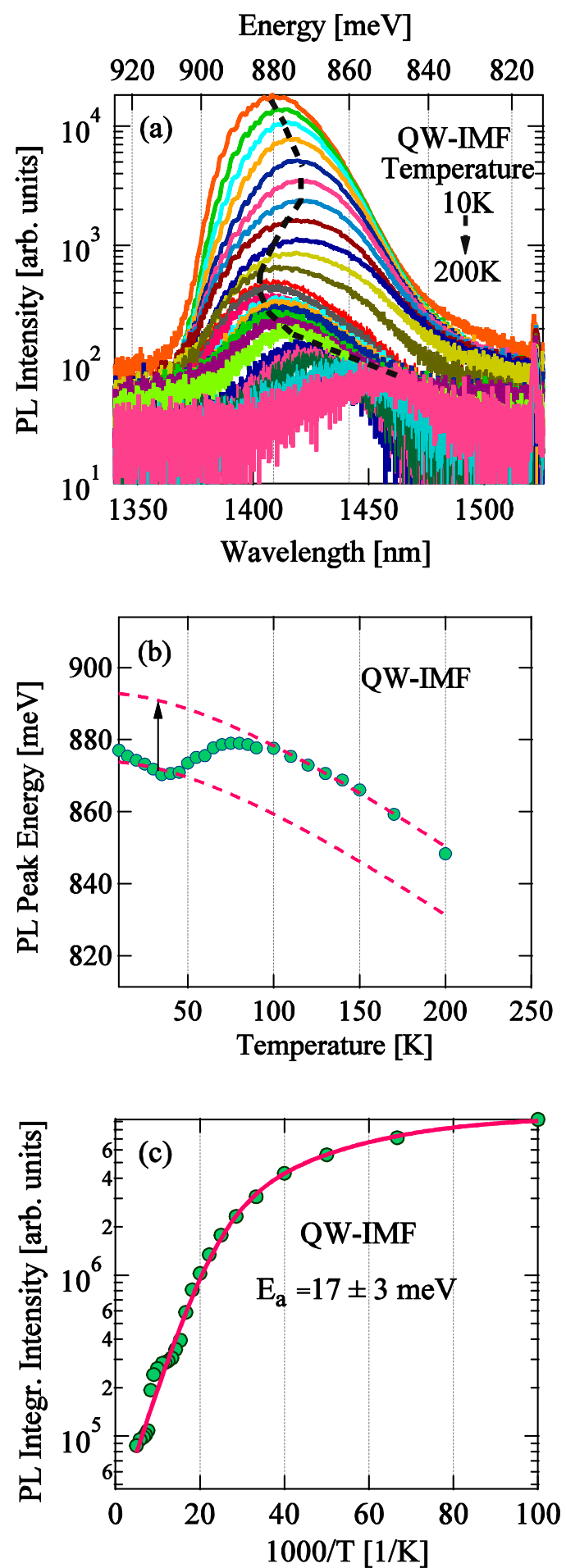
Figure 3 (1 col.)
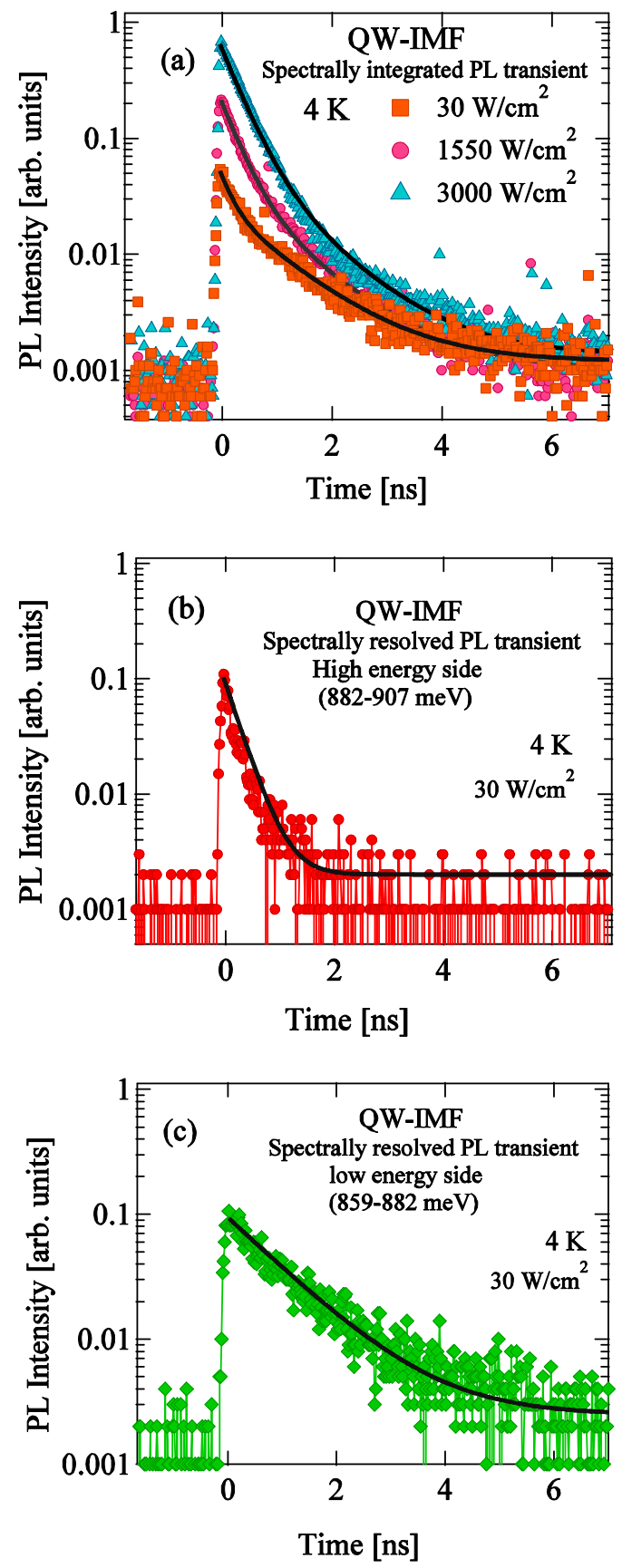
Figure 4 (1 col.)
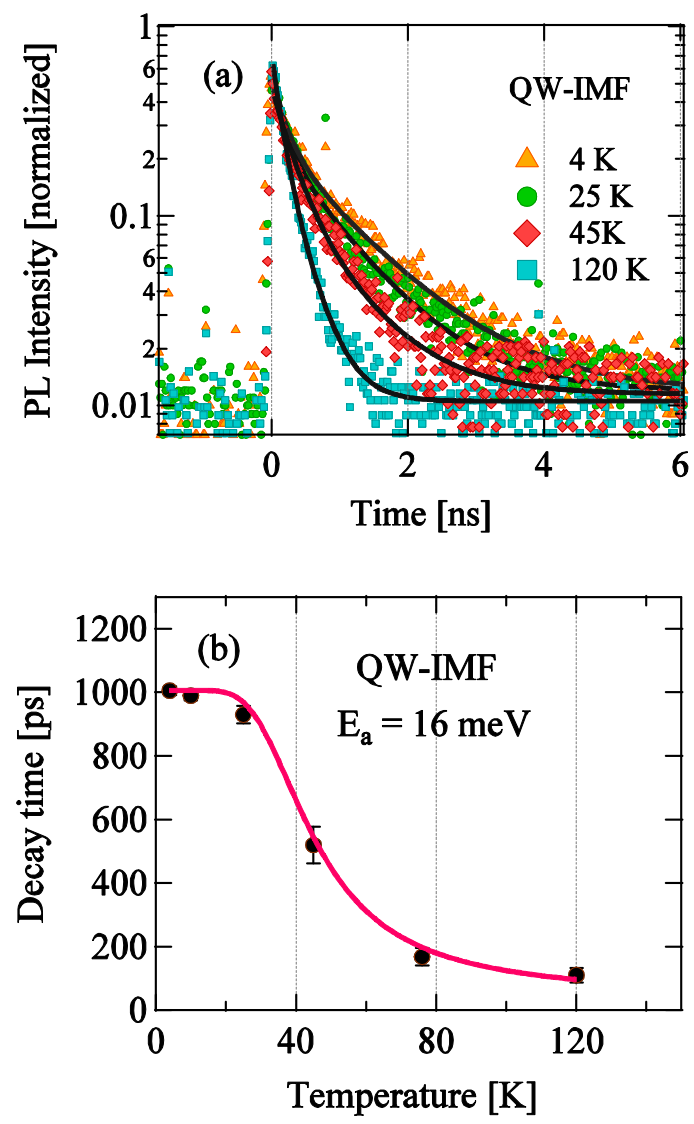
Figure 5 (1 col.)

Figure 6 (1 col.)

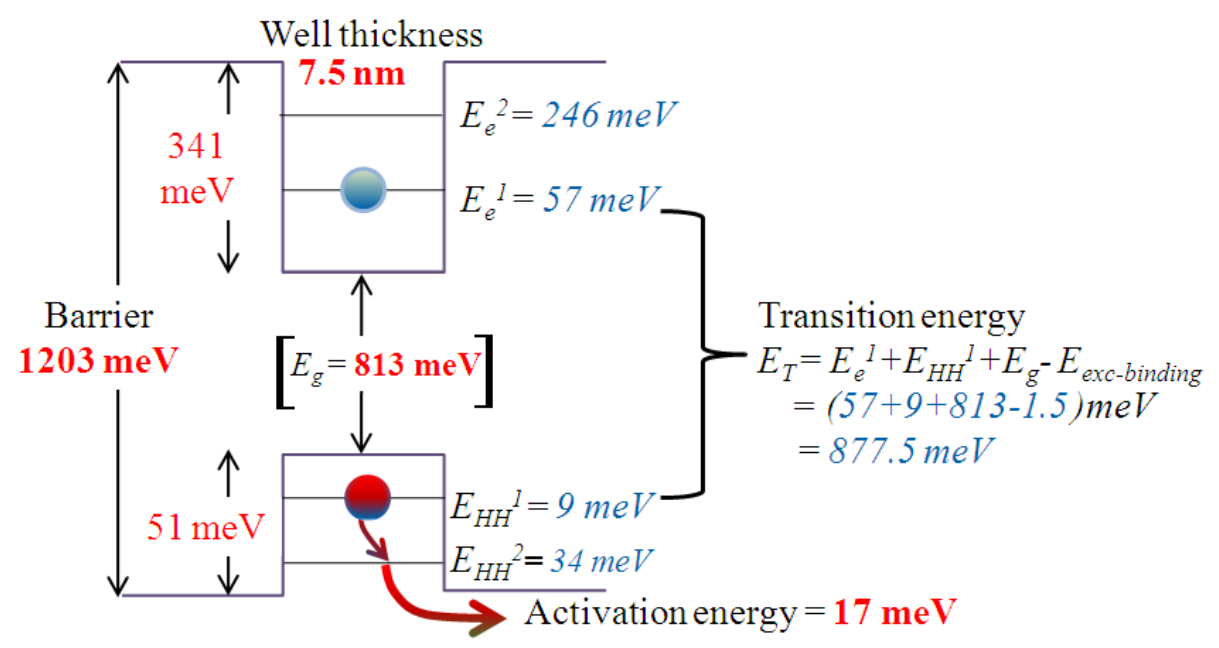

